\title{
Viscoelastic measurement techniques
}

\author{
R. S. Lakes ${ }^{\text {a) }}$ \\ Department of Engineering Physics, Engineering Mechanics Program, University of Wisconsin-Madison, \\ 147 Engineering Research Building, 1500 Engineering Drive, Madison, Wisconsin 53706-1687
}

(Received 23 July 2003; accepted 22 December 2003; published 8 March 2004)

\begin{abstract}
Methods for measuring viscoelastic properties of solids are reviewed. The nature of viscoelastic response is first presented. This is followed by a survey of time and frequency-domain considerations as they apply to mechanical measurements. Subresonant, resonant, and wave methods are discussed, with applications. (C) 2004 American Institute of Physics.
\end{abstract}

[DOI: 10.1063/1.1651639]

\section{INTRODUCTION}

\section{A. Principles of viscoelasticity}

\section{Measures of internal friction}

Viscoelastic materials have a relationship between stress and strain which depends on time or frequency. Anelastic solids represent a subset of viscoelastic materials: they have a unique equilibrium configuration and ultimately recover fully after removal of a transient load. Internal friction refers to the dissipative response of a material when subjected to a sinusoidal deformation. All materials exhibit viscoelastic response; elasticity or spring-like behavior does not exist in real materials but is an approximate description of materials for which the viscoelastic effects are small enough to ignore.

Viscoelasticity is of interest in the context of understanding physical processes such as molecular mobility in polymers, ${ }^{1}$ and of phase transformations, motion of defects, alloying atoms in crystalline solids. ${ }^{2}$ Viscoelasticity ${ }^{3}$ is also used in the design of materials and devices for a variety of purposes including earplugs, vibration abatement, reduction of mechanical shock, instrument mounts, and control of rebound and rolling resistance.

The loss angle $\delta$ is the phase angle between stress and strain during sinusoidal deformation in time. The loss angle or the loss tangent $\tan \delta$ as a measure of damping or internal friction in a linear material is advantageous in that it is clearly defined in terms of observable quantities. Tan $\delta$ depends on frequency, and it is customary to plot it on a logarithmic frequency scale. A factor ten ratio in frequency is referred to as a decade. Tan $\delta$ is also the ratio of the imaginary part $G^{\prime \prime}$ to the real part $G^{\prime}$ of the complex modulus $G^{*} \equiv G^{\prime}+i G^{\prime \prime}$. The quality factor $Q$ associated with the width of resonant peaks [see Eq. (3)] is given for small $\delta$ by $Q^{-1} \approx \tan \delta$. If vibration in a resonating system is allowed to decay with time due to material viscoelasticity after removal of the excitation, one may define the $\log$ decrement $\Lambda$ in terms of amplitudes $A_{1}$ and $A_{2}$ of successive cycles as $\Lambda$

${ }^{a)}$ Electronic mail: lakes@engr.wisc.edu $=\ln \left(A_{1} / A_{2}\right)$. For small $\delta, \Lambda \approx \pi \tan \delta$. The specific damping capacity $\Psi$ refers to the ratio ${ }^{1,4}$ of energy $\Delta W$ dissipated for a full cycle to the maximum elastic energy $W$ stored in the material. For a linear material, $\Psi=2 \pi \tan \delta$; but $\Psi$ is well defined even for nonlinear materials, consequently it is preferred by some authors. The material stiffness or modulus in viscoelastic materials depends on frequency. This is known as dispersion.

\section{Transient properties}

Transient properties are defined in terms of response to a step input in time. Creep refers to the time-dependent strain response to a step stress. The creep compliance is the strain divided by the constant stress, denoted $J_{E}(t)$ for a tension/ compression experiment, $J_{B}(t)$ for a bulk (volumetric) experiment, and $J_{G}(t)$ for a shear experiment. Stress relaxation refers to the time-dependent stress response to a step strain. The relaxation modulus is the stress divided by the constant strain. $G(t)$ is the relaxation modulus in shear, $E(t)$ in tension/compression, and $B(t)$ in bulk (volumetric) deformation. Creep and stress relaxation experiments can be done in tension, torsion, bending, bulk (volumetric), or other deformation modes. The time dependence of each of these will in general differ. These results are usually presented versus log time. The material is linear when $J(t)$ is independent of stress and $G(t)$ is independent of strain, otherwise it is nonlinear. Linearity of a material subjected to a series of creep tests at different stress can be displayed visually by plotting stress versus strain at a given creep time. Such a plot is called an isochronal; it is a straight line if the material is linearly viscoelastic. If the transient properties depend on the time after formation or transformation of the material as well as the time $t$ after load application, the material is said to exhibit physical aging.

\section{Significance. Relation to other relaxation processes}

Viscoelasticity in materials is studied since (i) viscoelastic solids are used to absorb vibration, (ii) viscoelastic effects 
are linked to physical processes such as phase transformations, diffusion, or motion of dislocations, vacancies and other defects, so that viscoelastic measurements are used as a probe into the physics of these processes, and (iii) viscoelasticity is relevant in phenomena such as ball rebound, rolling resistance, sag, droop, and spin instability. Also, viscoelastic relaxation in the mechanical properties may be related to relaxation processes in other material properties such as the dielectric permittivity, piezoelectric properties, magnetic properties, or thermal expansion.

\section{B. Overview of mechanical test principles}

\section{Load application and deformation measurement}

The simplest method of load application is to use a dead weight. This is appropriate for creep tests of moderate to long duration. Dynamic response may be achieved by generating force or torque via electromagnetic interaction between a coil carrying a controlled electric current and a permanent magnet. Larger forces and torques may be achieved via servocontrolled hydraulic systems. Such systems, typically capable of large forces, 40 to $400 \mathrm{kN}$, are commercially available.

\section{Temperature}

Since viscoelastic properties depend on temperature, sometimes sensitively, environmental control of temperature is usually used. Polymers in the vicinity of the glass transition and crystalline materials in the vicinity of a phase transformation are very sensitive to temperature, so it is usual to control temperature within $0.1{ }^{\circ} \mathrm{C}$ or better. In tests in tension/compression, a length change can arise from creep or from thermal expansion. Therefore excellent temperature control is required in tensile creep or relaxation studies. Thermal expansion of an isotropic material has no shear or torsional component, therefore torsion tests are less demanding of accurate temperature control than are tension or compression tests. Many polymers as well as concrete and biological materials are sensitive to hydration, therefore humidity or saturation is monitored or controlled in such tests. Testing at elevated temperatures presents a variety of challenges. Since most transducers for load and deformation do not survive high-temperature conditions, it is typical to isolate the heated specimen from other parts of the apparatus. Examples are given in Sec. III B.

\section{Interpretation}

Interpretation of tests is based on use of an analytical solution for the deformation field in the specimen geometry in question. If a specimen is gripped at the ends, the details of the grip stress may not be well known, therefore tension, torsion, or bending tests on gripped specimens are usually done on long, slender specimens with length at least ten times the diameter. The reason is that the nonuniform stress at the ends decays over a distance of one or two diameters and has minimal influence on the results for a long specimen. The slenderness requirement is more severe if the specimen is anisotropic.

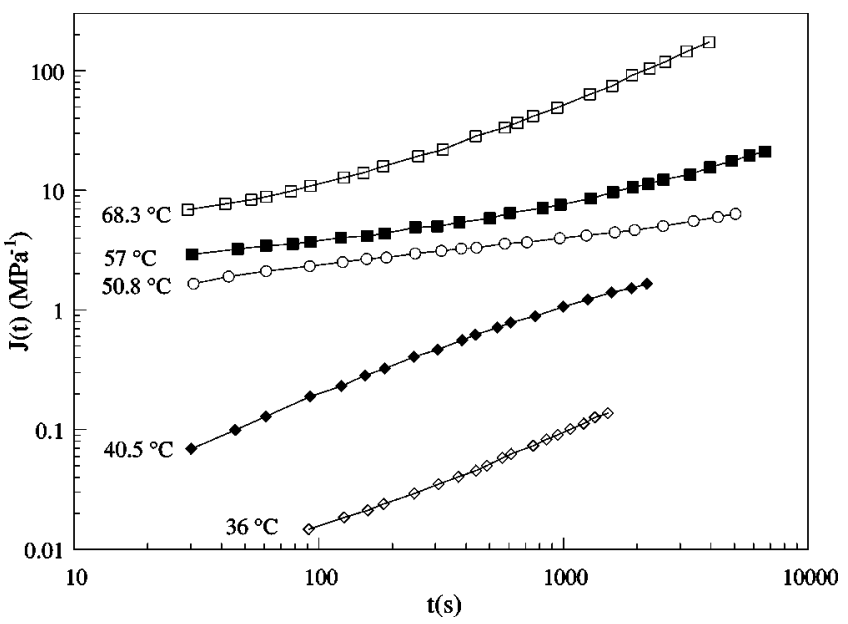

FIG. 1. Creep compliance $J(t)$ of polyvinyl acetates at various temperatures, adapted from Ref. 6.

\section{Outline of the review}

Within our scope we will consider methods for measuring viscoelastic properties of solid materials. Section II deals with time-domain transient properties: creep and stress relaxation. Section III treats material response to sinusoidal input under subresonant (quasistatic) conditions in which inertial terms are negligible. Methods for phase measurement are considered, as are several kinds of instrument. Section IV deals with sinusoidal input measurement near the resonant frequencies of a specimen. For both resonant and subresonant conditions, methods are presented for handling large and small phase angle between stress and strain. Section V introduces variants of the methodology to make measurements on the microscale and nanoscale. Section VI introduces wave methods. Section VII deals with the challenge of obtaining a wide window on the time or frequency domain.

\section{TIME DOMAIN MEASUREMENTS}

\section{A. Creep}

Creep is one of the simplest experimental modalities for characterizing viscoelastic behavior. A dead weight can provide constant stress, provided the deformation is sufficiently small that the specimen cross section does not change appreciably. The load history in time cannot follow a mathematical step function as one would prefer based on the definition of the creep compliance. Because the rise time is not zero, one ordinarily begins taking data after a period about ten times as long as the rise time has elapsed..$^{5}$ It is difficult to apply a dead weight in less than 0.5 to $1 \mathrm{~s}$, therefore such creep data begin at 5 to $10 \mathrm{~s}$. A test from $10 \mathrm{~s}$ to $3 \mathrm{~h}$ covers three decades; $28 \mathrm{~h}$ is required for four decades. Typical creep results $^{6}$ are shown in Fig. 1. The upper limit on the length of time of a creep test is limited by the experimenter's patience. In some cases in which long-term data are required but cannot be inferred from time-temperature shifts (see Sec. VII), test time can be up to 20 years. $^{7}$

Prior to testing, one should monitor deformation at zero load for a period of time to make sure strain from specimen preparation has recovered. Moreover, prior to testing, poly- 


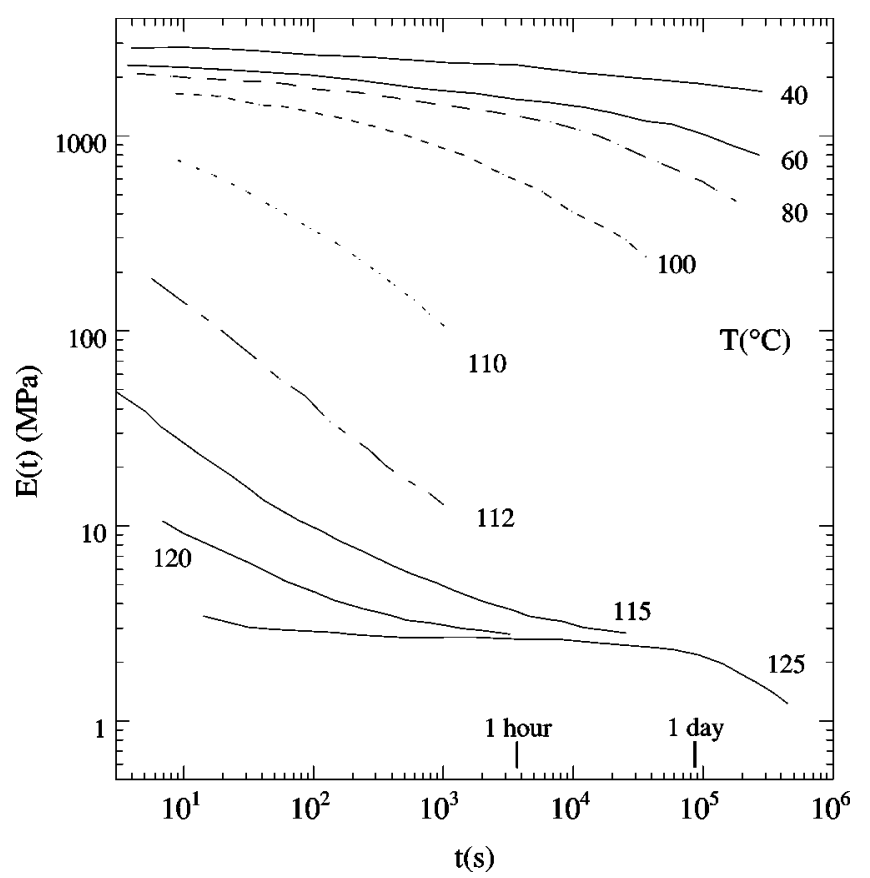

FIG. 2. Relaxation modulus E(t) of PMMA at various temperatures, adapted from McLoughlin and Tobolsky. ${ }^{10}$

mer specimens are generally preconditioned by applying a few load cycles consisting of creep and recovery segments. This is done to achieve reproducible results. ${ }^{8}$ Biological materials exhibit similar effects.

As for instruments, the torsion-pendulum implementations for subresonant tests (Sec. III B) can be used for creep studies.

\section{B. Stress relaxation}

Stress relaxation involves observation of time-dependent stress in response to a step strain. The step strain can be achieved by using a cam system that is much stiffer than the specimen to impose a constant deformation following a risetime transient, or by manually advancing a screw up to a stop. For example a relaxation instrument for the study of glassy polymers used an eccentric cam system actuated by a handle for load application ${ }^{9}$ to achieve a range in time of three decades. An axial strain-gauge-type load cell was used for force measurement. Because in stress relaxation the specimen deformation is to be held constant, it is necessary to determine the compliance of the load cell, to verify that it (and other parts of the test frame) was much stiffer than any specimen studied. For tension experiments both stressinduced deformation and thermal expansion of the load frame can introduce error. The load frame must therefore be made robust, and should be enclosed in a temperaturecontrolled chamber independent of the temperature-control system for the specimen. Since polymers can swell with changes in humidity, it is necessary to accurately control humidity in tensile experiments. Results for polymethyl methacrylate (PMMA) ${ }^{10}$ at several temperatures are shown in Fig. 2. Alternatively a step strain in time can be applied via a servocontrol device in which strain or displacement is used as a feedback signal.

\section{FREQUENCY DOMAIN: SUBRESONANT}

\section{A. Phase measurement}

For a load history which is sinusoidal in time, the deformation history is also sinusoidal in time, with a phase shift, provided the material is linearly viscoelastic and the apparatus is linear. This review is for the most part limited to linear systems. For nonlinear materials or devices, overtones (multiples) of the driving frequency appear in the deformation. At frequencies sufficiently far below the lowest natural frequency that may be excited in the specimen or in the transducers, or in other parts of the apparatus, the phase angle between load and deformation (or between torque and angular displacement) is essentially equal to the phase angle $\delta$ between stress and strain. The driving frequency may be tuned or scanned over a range. ${ }^{11}$ The lower limit of this range is limited by drift in the electronics and principally by the experimenter's patience. The upper limit of the frequency range is limited by resonances in the specimen or in parts of the apparatus. In the following, the material is assumed to be linearly viscoelastic, so if the stress is sinusoidal in time, the strain is also sinusoidal, with a phase shift. As with other mechanical measurements, one must determine the compliance of the instrument to make sure the resulting errors in the calculated material modulus are sufficiently small. In addition, phase error due to amplifiers and other electronics must be quantified so that the investigator is confident the measured phase is representative of the material phase $\delta$. Moreover, instrumental compliance can contribute a phase error even at frequencies well below any natural frequency. ${ }^{12}$

\section{Time delay}

Measurement of the phase $\delta$ can be performed by determining the time delay $\Delta t$ between the sinusoids. The relationship is $\Delta t=T \delta / 2 \pi$, with $T$ as the period of the sinusoid. The time delay can be determined either on an oscilloscope or by detecting the zero-crossing of the load and displacement signals and measuring the time delay with a high-speed timer. Noise of electrical or other origin limits the resolution of the phase.

\section{Lissajous $(x-y)$ figures}

A graph of load versus deformation (sinusoidal in time) for a linearly viscoelastic material is elliptic in shape (Fig. $3)$. The phase $\delta$ is given by

$$
\sin \delta=A / B
$$

with $A$ as the horizontal thickness of the ellipse ${ }^{3}$ and $B$ as the full width of the figure. To measure small phase angles, the middle of the ellipse can be magnified on an oscilloscope or computer display. Again, the ratio of signal to noise must be sufficiently high to resolve the phase. The area within the figure is representative of the energy dissipated per cycle in the material.

\section{Lock-in measurement}

Lock-in amplifiers, given a reference source at a known frequency, amplify only at that frequency. They are therefore capable of accurate measurements in the presence of noise, 


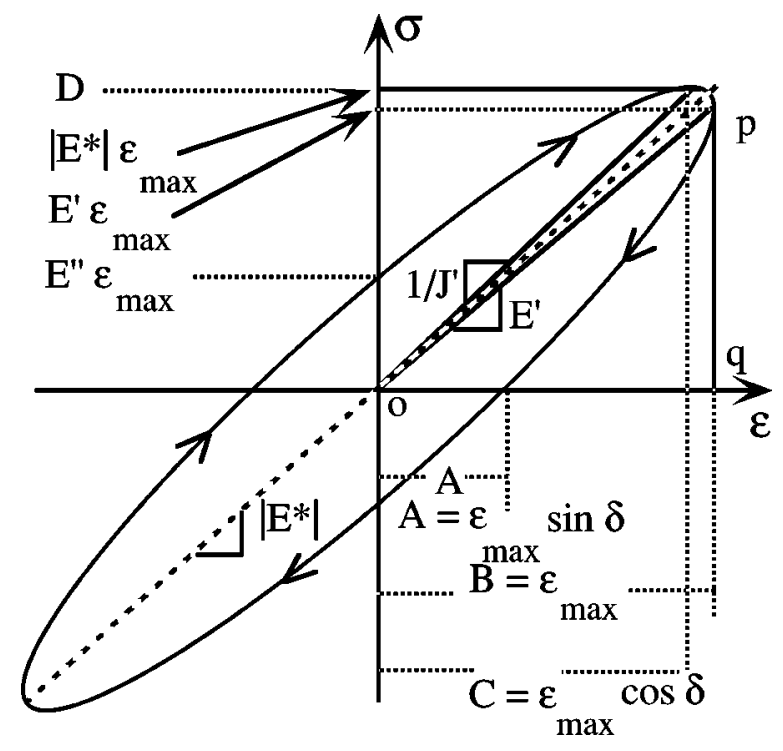

FIG. 3. Lissajous figure due to strain $(\epsilon)$ response to stress $(\sigma)$ that is sinusoidal in time, in relation to phase angle $\delta$, between stress and strain after Lakes. ${ }^{3} E^{*} \equiv E^{\prime}+i E^{\prime \prime}$ is the complex modulus.

which is distributed over a range of frequency. A two-phase lock-in amplifier provides phase information as well, and is therefore suitable for viscoelastic measurements. Phase measurement with a lock-in amplifier reduces the scatter inherent in the determination of phase. The typical frequency range is $1 \mathrm{~Hz}$ to $100 \mathrm{kHz}$, but some models go as low as $0.001 \mathrm{~Hz}$ or as high as $200 \mathrm{MHz}$. Phase resolution of some models is claimed to be $0.001^{\circ}\left(\delta=1.8 \times 10^{-5}\right)$ however the actual resolution will depend on the levels of noise and on instrument settings, especially the time constant. Automated scans of frequency are possible with some lock-in amplifiers.

\section{B. Instruments}

Commercial servohydraulic test machines make use of an electrical feedback signal from a force transducer, displacement transducer, or strain gauge to control a hydraulic valve. This valve governs the flow of hydraulic fluid that, by pressing on a piston, provides the force. These machines are usually used to perform stress-strain tests to fracture; they can also be used for creep or stress-relaxation measurements, or subresonant tests at low frequency, typically below $10 \mathrm{~Hz}$. Instrumental resonances limit the frequency range of these devices.

Commercial "dynamic mechanical analyzers" typically use a servocontrolled electromagnetic driver to apply a flexural load to a bar specimen. Creep, relaxation, and low frequency subresonant sinusoidal tests can be done with these devices. Instrumental resonances usually limit the frequency range to below $10 \mathrm{~Hz}$. The range of time or frequency can be extended by conducting repeated tests at several temperatures and using time-temperature superposition (Sec. VII B) to infer viscoelastic properties over a wider range. This approach is applicable only to materials dominated by a single relaxation mechanism.

Torsion pendulums (Fig. 4), which have been made in many variants, typically based on the classical Kê

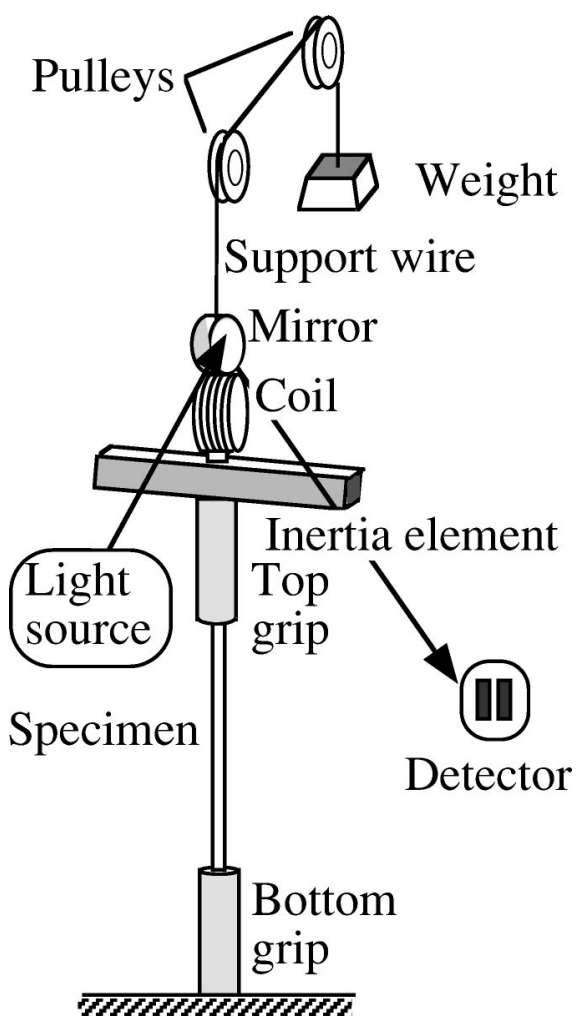

FIG. 4. Torsion pendulum of inverted type. Torque is generated by the action of a magnetic field supplied by a permanent magnet (not shown) upon the coil.

pendulum, ${ }^{13}$ are described in more detail in Sec. IV A. They can be used for creep and for driven sinusoidal tests in the subresonant domain provided one has sufficient precision in the measurement of phase. In a typical embodiment, electromagnetic drive is used to apply a torque. In earlier instruments a moving coil was placed in the field of a permanent magnet. With the advent of better magnet materials, it is now more practical to use a moving magnet in the field of a coil to avoid concern of error caused by stiffness and damping caused by electrical wires supplying a moving coil. An optical lever is used to determine angular displacement. Early instruments made use of a beam of light projected on a scale and observed visually; more recent ones use a laser beam and a split silicon sensor. Torsion offers the advantage that small deformation is readily measured.

A high-resolution inverted torsion pendulum ${ }^{14}$ (Fig. 5) provides a six-decade range of frequency from $10^{-5}$ to 10 $\mathrm{Hz}$ under subresonant conditions below the natural frequency of 100 to $500 \mathrm{~Hz}$. Phase is determined by using a timer to measure the time delay between the zero-crossing of the load and displacement signals. A phase uncertainty $\delta \phi \approx 6$ $\times 10^{-5}$ at $10 \mathrm{~Hz}$, and better at lower frequencies, was claimed, based on a time accuracy of $1 \mu \mathrm{s}$. To achieve such phase resolution it is necessary to minimize noise sources of electrical and mechanical origin. The temperature range is room temperature to $800^{\circ} \mathrm{C}$, under vacuum. Representative results are shown in Fig. 6.

Low-temperature variants ${ }^{15}$ have been used to study superconductors at cryogenic temperature $(4.7 \mathrm{~K})$. As with the above device, the specimen is kept isolated from the drive 


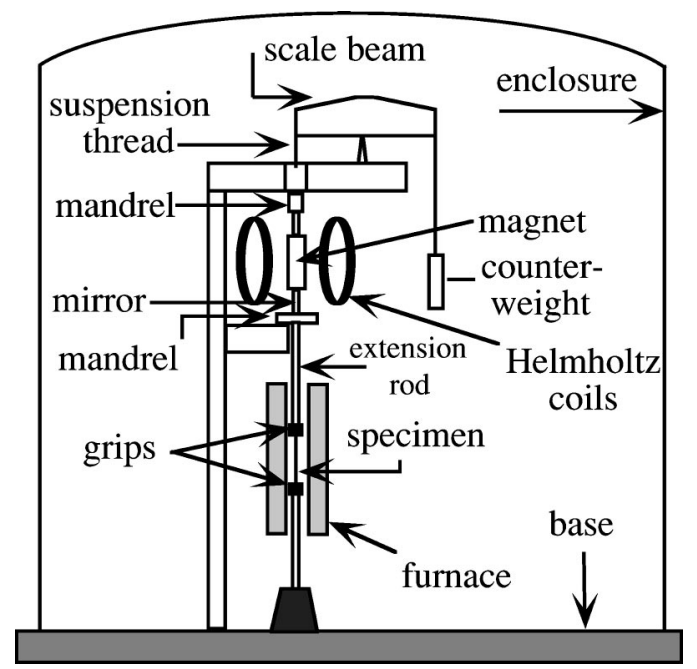

FIG. 5. Driven subresonant torsion pendulum, adapted from Woirgard et al. ${ }^{14}$

system and twist-measurement system by an arrangement of stalks. These permit the specimen to be cooled in a cryostat and also to be subjected to a magnetic field up to $0.2 \mathrm{~T}$ (2000 $\mathrm{G})$, but limit the frequency to below $10 \mathrm{~Hz}$. In this system both the temperature and magnetic field near the specimen are isolated from the magnetic-drive system.

High-temperature variants ${ }^{16}$ allow measurements to $1800 \mathrm{~K}$. In this device, data are obtained by comparison of the deformation of the specimen (in a furnace) with the deformation of an elastic standard (outside the furnace). An instrument $^{17}$ for the study of creep and internal friction of geological materials up to $1400{ }^{\circ} \mathrm{C}$ makes use of a refractory molybdenum alloy in the high-temperature section. The specimen is gripped with the aid of a mismatch in thermal expansion between specimen and refractory grips. The heated specimen is separated by connecting stalks and a base plate from a water-cooled low-temperature zone containing the transducers: a brushless motor to generate the torque, a custom torque gage based on strain gauges, and two noncontacting inductive displacement transducers to determine the

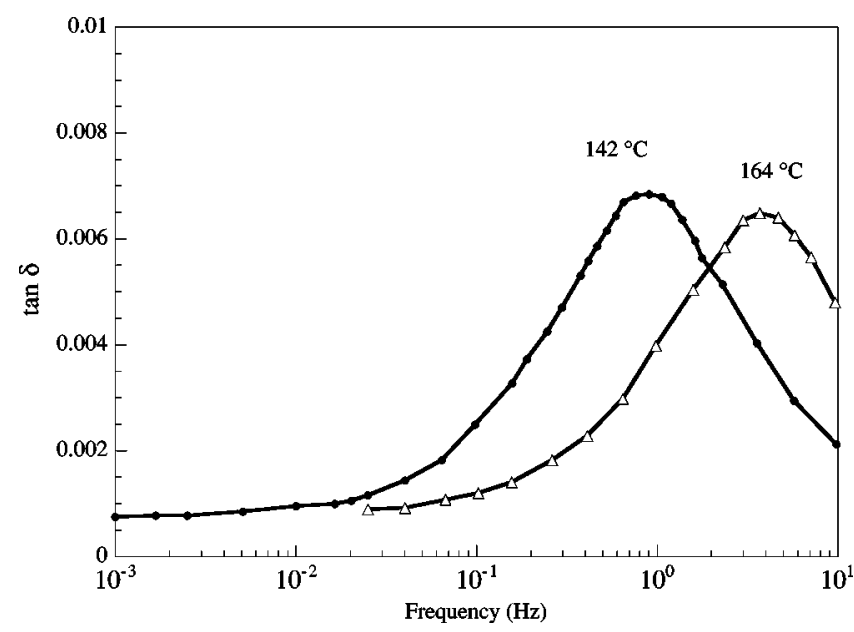

FIG. 6. Mechanical damping $\tan \delta$ of niobium vs frequency in the subresonant regime, showing a Snoek peak due to interstitial oxygen. Adapted from Ref. 14.

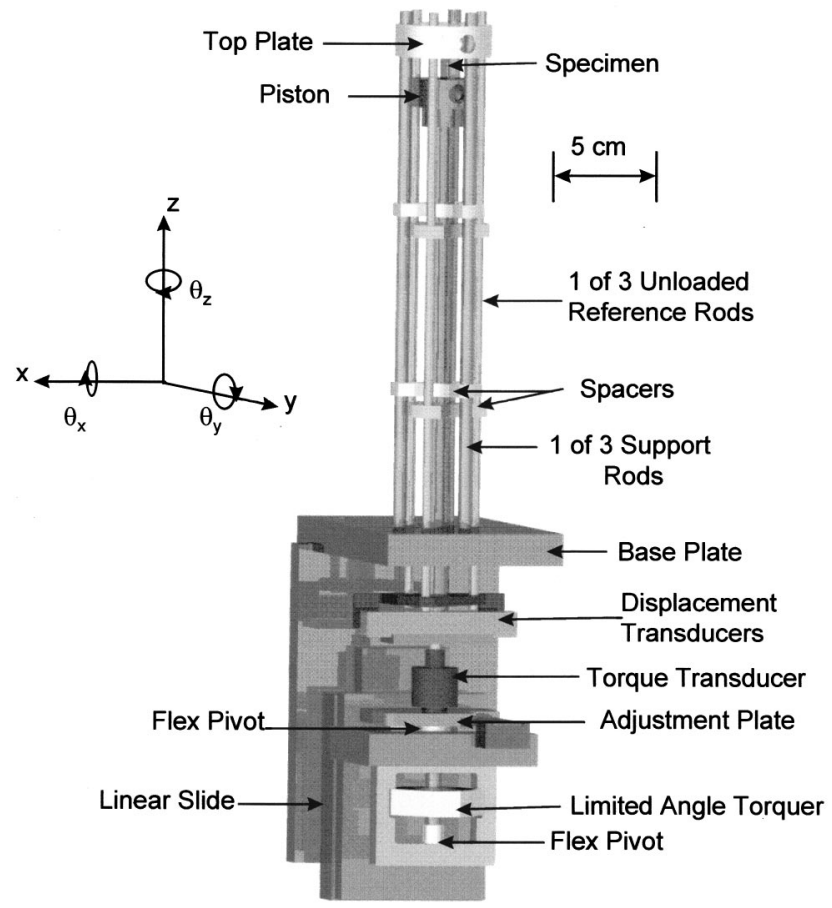

FIG. 7. Driven high-temperature subresonant torsion pendulum, adapted from Gribb and Cooper. ${ }^{17}$ High-temperature components are made from a refractory molybdenum alloy. The apparatus is enclosed in a gas-tight chamber, and the upper portion is sealed by an O-ring.

angular displacement (Fig. 7). The stalks and other parts necessary to isolate the heated specimen give rise to an instrumental fundamental resonance of $100 \mathrm{~Hz}$. Since the seismic frequency range of interest is 0.001 to $1 \mathrm{~Hz}$, the frequency range in the subresonant regime of the instrument is adequate.

A variant suitable for a broad band of frequency, including subresonant and resonant, is described in Sec. VII D.

\section{Treatment of large and small phase}

For materials of low loss $\left(\tan \delta<10^{-3}\right)$, resonance methods (Sec. IV) are the most appropriate because they are sensitive. By contrast small phase differences are obscured by noise and are difficult to measure directly. If it is necessary to make subresonant measurements on low-loss solids, excellent phase resolution is required. Phase resolution may be limited by the ratio of signal to noise. A lock-in amplifier can be beneficial in cutting through the noise and improving phase resolution. Moreover one must reduce phase errors that may be introduced by transducers, electronics, and filters.

Large phase angles are easy to measure. If, however, the material tends to a fluid consistency at sufficiently low frequency or long time, the specimen may not be able to support its own weight. Methods for viscoelastic fluids are outside the scope of this review. 


\section{FREQUENCY DOMAIN: RESONANT}

\section{A. Lumped resonances: pendulum devices}

Resonance methods are appropriate for low-loss materials with $\tan \delta$ from $10^{-3}$ to $10^{-9}$ or smaller. By contrast, in the subresonant domain small $\tan \delta$ values correspond to small phase angles, which are difficult to measure.

The specimen is usually a long bar or rod with an attached mass much greater than that of the specimen. This is referred to as a lumped system since virtually all the inertia is associated with the attached mass, and virtually all the elastic or viscoelastic compliance is associated with the specimen. Analysis of the response of such a system is simple.

Consider a torsion pendulum with a heavy member of mass moment of inertia $I$ at the end of the specimen which has shear modulus $G$ and damping (phase $\delta$ between stress and strain). If the specimen itself supports the inertia member, then it is under a static tension force that could influence the torsion results or cause creep in high temperature tests. To avoid this, it is common to use an inverted torsion pendulum with the specimen below the inertia member, the weight of which is supported by a fine wire of negligible torsional stiffness in comparison with the specimen. The support wire is strong and is sufficiently slender so that its torsional stiffness does not significantly perturb the measurement. In these devices the specimen, mounted below the inertia member, experiences pure torsion with no tension. A typical embodiment of the idea is shown in Fig. 4. The torsion pendulum is normally operated at a low resonant frequency, e.g., $1 \mathrm{~Hz}$, and the temperature scanned. There are many examples of this approach. ${ }^{11}$ The original Kê pendulum $^{13}$ used a galvanometer-coil winding in a static magnetic field to generate the torque and light reflected from a mirror to determine the angular displacement. It was used for creep and for determination of internal friction by free decay of vibration (described below).

Measurement of the natural frequency $\nu_{1}$ allows one to calculate $^{3,18}$ the shear modulus as follows:

$$
\nu_{1}=\sqrt{\frac{G^{\prime}\left(\nu_{1}\right) k}{I}} .
$$

For a round, straight rod, $k=\pi d^{4} / 32 L$, with $d$ as the rod diameter and $L$ as the rod length; $I$ is the mass moment of inertia of the attached mass. The natural frequency can be determined by observing the period of free vibrations following a perturbation or by tuning the frequency in a driven (forced) system.

The damping $\tan \delta$, provided it is small, is calculated from the full width $\Delta \nu$ of the resonance curve at halfmaximum amplitude

$$
\frac{\Delta \nu}{\nu_{1}} \approx \sqrt{3} \tan \delta
$$

This measurement of the peak width gives "quality factor" $Q$, with $Q^{-1} \approx \tan \delta$ for small $\delta$.

In a driven system one can make measurements away from the resonant peak. To interpret, observe that the phase angle $\phi$ between torque and angular displacement for a lumped system of one degree of freedom is given by

$$
\tan \phi=\frac{\tan \delta}{1-\left(\nu / \nu_{1}\right)^{2}} .
$$

The phase angle $\phi$ is approximately the same as $\delta$ for sufficiently low frequency $\nu$ below the natural frequency $\nu_{1}, \pi / 2$ radians at $\nu_{1}$, and $\pi$ radians for high frequency. The relationship between structural stiffness and material stiffness (modulus) for this lumped system is, with $\theta_{0}$ as the angular displacement amplitude and $\mathrm{M}_{0}$ as the applied moment amplitude

$$
\frac{G^{\prime}(\nu) k \theta_{0}}{M_{0}}=\sqrt{\frac{1}{\left[1-\left(\nu / \nu_{1}\right)^{2}\right]^{2}+\tan ^{2} \delta}} .
$$

For a round, straight rod, $k=\pi d^{4} / 32 L$, with $d$ as the rod diameter and $L$ as the rod length. If the actual experimental instrument has stalks and heavy attached transducers, its resonance structure will not be so simple; if instrument resonances are at frequencies close to or lower than specimen resonances, the instrument is generally suitable only for subresonant studies.

One can also obtain the damping $\tan \delta$, provided it is small, from the free-decay time $t_{1 / e}$ of vibration (of period $\left.T=1 / \nu_{1}\right)$ to decrease by a factor $e=2.718$ following an impulse

$$
\tan \delta \approx \frac{1}{\pi} \frac{T}{\mathrm{t}_{1 / e}} .
$$

The free-decay approach offers simplicity in that no tunable function generator is needed; it is sufficient to apply a perturbation. If the torsion pendulum has a sufficiently massive inertia member, the natural frequency will be on the order 1 $\mathrm{Hz}$, and the perturbation can be applied manually, by a mechanical system, or electrically. One may also speak of the $\log$ decrement $\Lambda$ in terms of amplitudes $A_{1}$ and $A_{2}$ of successive cycles as $\Lambda=\ln \left(A_{1} / A_{2}\right)$. For small $\delta, \Lambda \approx \pi \tan \delta$. Classic results are shown in Fig. 8.

In lumped-resonance procedures it is usual to scan the temperature while observing the behavior near resonance. One can also vary the frequency by varying the attached inertia, but this is cumbersome.

\section{B. Distributed long-bar resonances}

Long bars are used for resonant experiments because they have a relatively simple resonance structure, which simplifies interpretation of results. Resonance measurements have been recognized following Förster's 1937 pioneering work. ${ }^{19}$ One may excite vibrations in extension, flexure, or torsion. The frequencies are as follows, assuming negligible added mass at the end, with $E$ as Young's modulus, $G$ as the shear modulus, and $\rho$ as density, for a bar fixed at one end, free at the other. Here the bar length $L$ is one-quarter wavelength. This is in contrast to a bar free at both ends for which the bar length is one-half wavelength. 


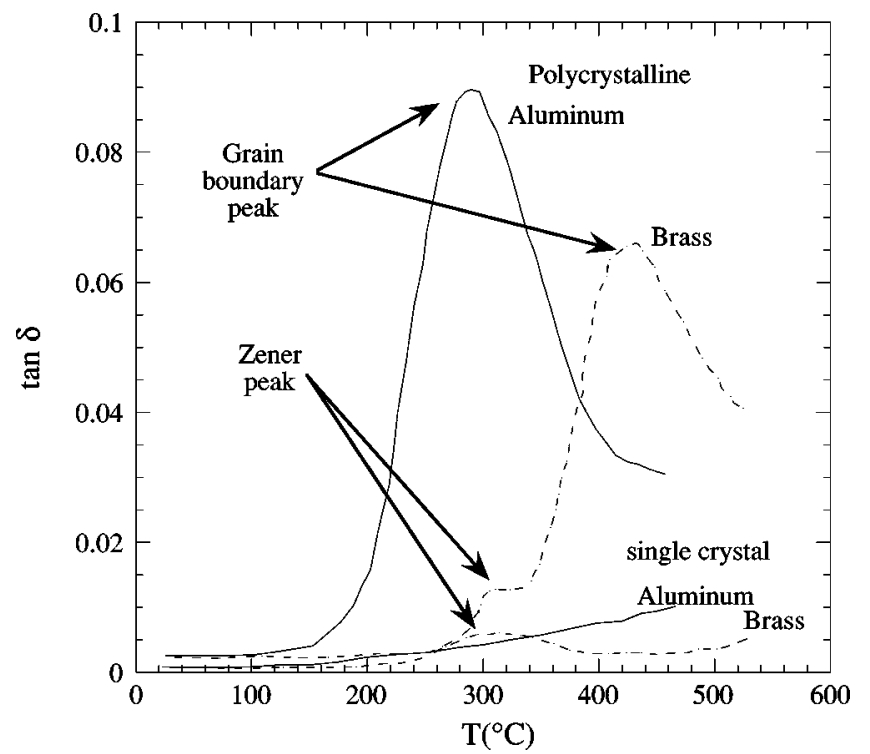

FIG. 8. Tan $\delta$ vs temperature for aluminum ${ }^{13}$ and brass $^{76}$ at low frequency, adapted from Kê.

$$
\nu_{n}=\frac{n}{4 L} \sqrt{\frac{E}{\rho}},
$$

for axial deformation assuming a wavelength much longer than the specimen width, ${ }^{2}$

$$
\nu_{n}=\frac{n}{4 L} \sqrt{\frac{G}{\rho}},
$$

for torsion, and

$$
\nu_{n}=\frac{h}{L^{2}} \frac{\alpha_{n}^{2}}{4 \sqrt{ } 3 \pi} \sqrt{\frac{E}{\rho}},
$$

for flexure 20,21 of a strip of length $L$ and thickness $h$, with $\alpha_{1}=1.875, \alpha_{2}=4.694$, and $\alpha_{3}=7.855$. Here $\sqrt{\mathrm{E} / \rho}$ is the speed of longitudinal bar waves and $\sqrt{\mathrm{G} / \rho}$ is the speed of torsional waves. Material damping is determined via Eq. (3), which applies to distributed systems as well as lumped ones, provided $\tan \delta$ is sufficiently small (less than 0.1). As for flexure, many devices have been designed. The vibratingreed approach is particularly convenient. One end of a bar is clamped and the free end is mechanically excited. Capacitive excitation allows one to generate vibration without adding any mass to the specimen. Since the coupling is weak, the approach is suitable for materials of low damping. Figure 9

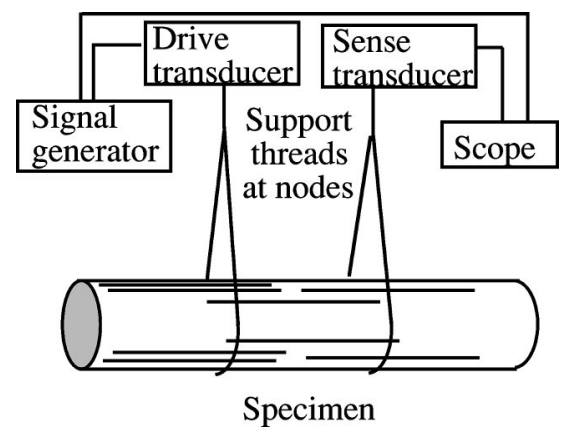

FIG. 9. Free-free flexure-resonance apparatus.

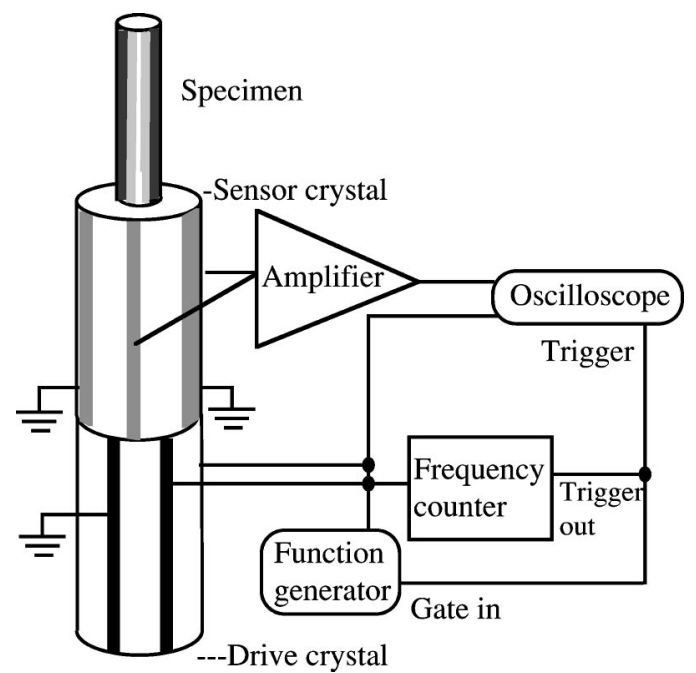

FIG. 10. Piezoelectric ultrasonic composite oscillator apparatus.

shows a typical setup for free-free vibration in which the specimen is free at both ends.

\section{Piezoelectric ultrasonic oscillator}

The piezoelectric ultrasonic composite oscillator technique is based on a device that consists of two piezoelectric crystals and a specimen cemented together ${ }^{22-24}$ (Fig. 10). The vibration may be in tension/compression or in torsion depending on the type and orientation of crystal. The resonant frequencies $\nu_{n}=c / \lambda=c(n+1 / 2) / L$ are governed by the length $L$ of the crystals and the speed $c$ of the ultrasonic waves in them. Here $n$ is an integer; the fundamental corresponds to $n=0$, and a wavelength twice the length, corresponding to the free-free boundary condition of a bar free at both ends. The specimen is regarded as effectively free even at the glued end because the specimen length is chosen so its natural frequency matches that of the crystals, so that the stress across the glued interface is minimal. Frequencies from 20 to about $120 \mathrm{kHz}$ are typical. Quartz is preferred for the crystals since it has a low $\tan \delta$, which can be below $10^{-6}$ depending on crystal defects. One crystal is driven electrically to induce vibration. The oscillating voltage induced by strain in the other crystal is measured. The wires are attached to the crystals' conducting metal plating at the crystal midpoints, which are nodes for free-free vibration. Because the wire has nonzero size, there is a parasitic damping associated with it. There is also a parasitic damping due to the cement used to attach crystal and specimen. This damping is small provided each crystal as well as the specimen has nearly the same natural frequency in which case the stress at the glue joint is minimal. The crystal itself has some damping which is accounted for in the calculation of specimen damping.

Viscoelastic properties of the specimen are inferred as follows from electrical measurements upon the sensor crystal and from the dimensions and masses of specimen and crystals:

$$
\begin{aligned}
\tan \delta_{\mathrm{t}}= & \left(m_{1} a_{1}^{2} \tan \delta_{1}+m_{2} a_{2}^{2} \tan \delta_{2}+m_{s p} a_{s p}^{2} \tan \delta_{s p}\right) \\
& /\left(m_{1} a_{1}^{2}+m_{2} a_{2}^{2}+m_{s p} a_{s p}^{2}\right)
\end{aligned}
$$




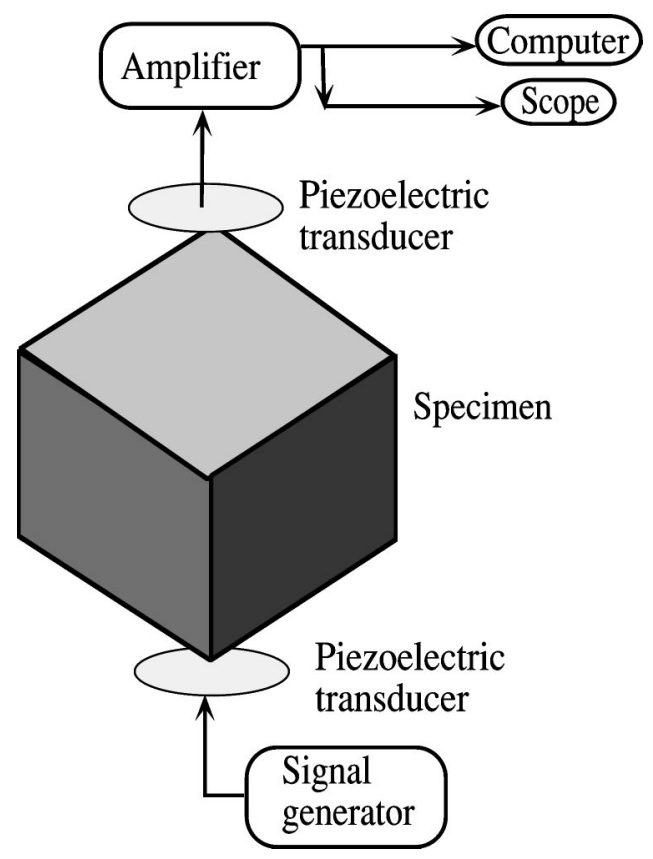

FIG. 11. Resonant ultrasound spectroscopy (RUS) apparatus.

$$
\begin{aligned}
\omega_{t}^{2}= & \left(m_{1} a_{1}^{2} \omega_{1}^{2}+m_{2} a_{2}^{2} \omega_{2}^{2}+m_{s p} a_{s p}^{2} \omega_{s p}^{2}\right) \\
& /\left(m_{1} a_{1}^{2}+m_{2} a_{2}^{2}+m_{s p} a_{s p}^{2}\right)
\end{aligned}
$$

in which $\omega$ is resonant angular frequency, $m$ is mass, and $a$ is radius, and subscripts 1 and 2 refer to the crystals and sp to the specimen. ${ }^{25}$ It is assumed that the electrical impedance of the circuit attached to the gauge (sensor) crystal is sufficiently high that energy losses associated with it can be neglected.

The method is usually used to measure modulus and damping at a constant frequency over a range of temperature. If the intended temperature exceeds the Curie point of crystal quartz, a long stalk of fused quartz (of length chosen to match the crystal frequency) is used between specimen and crystal so that the crystals can operate at a lower temperature. In addition to the fundamental natural frequency, one can also excite higher harmonics, however they are weaker in amplitude than the fundamental.

\section{Resonant ultrasound spectroscopy (RUS)}

Resonant ultrasound spectroscopy (RUS) measures of the resonance structure of a compact specimen such as a cube, parallelepiped, sphere, or short cylinder ${ }^{25,26,27}$ to infer material properties. Elastic moduli or complex dynamic viscoelastic moduli are determined from the resonant frequencies. Viscoelastic damping is determined from resonant-peak width following Eq. (3). Frequencies are typically from 50 $\mathrm{kHz}$ to $20 \mathrm{MHz}$ depending on specimen size and properties. A cubical specimen is typically held by contact force between piezoelectric ultrasonic transducers at opposite corners (Fig. 11). A short cylinder may be held between opposite edges. One transducer is a transmitter, and one is a receiver. Corners are used since they provide elastically weak coupling to the transducers, hence minimal perturbation to the vibration, so minimum parasitic damping. RUS requires no gluing, clamping, or painstaking alignment of the specimen. In comparison with pulsed wave ultrasound (Sec. VI), RUS requires no corrections for diffraction or concern about plane-wave approximations. Although RUS is conceptually and experimentally simple, determination of anisotropic elastic constants entails elaborate numerical methods.

All the elastic moduli $C_{i j k l}$ of a single specimen can be obtained from a single frequency scan. A numerical algorithm is used to extract the moduli from the resonant frequencies. A rectangular-parallelepiped specimen with its axes aligned with the material symmetry is commonly used. For the higher symmetries it is desirable to have unequal edge lengths to avoid overlap (degeneracy) of different modes with the same frequency; the algorithm cannot handle this overlap. The specimen must be accurately cut. Any error in parallelism or dimensions will translate into a similar size error in the moduli. A poorly cut specimen can prevent the numerical inversion from accurately functioning since then the assumed shape does not correspond to the actual shape. Most resonant frequencies in RUS depend on combinations of elastic constants. ${ }^{27}$ Numerical determination of anisotropic moduli using currently available software requires a good initial guess as input to the algorithm, since it is usually not straightforward to identify the modes. Since the lowest modes depend primarily on the principal shear modulus, 10 to 40 modes may be used in study of anisotropic solids. Missing modes can occur if the specimen displacement at the contact point is zero or is in a direction to which the transducer is not sensitive. Misidentified modes play havoc with numerical inversion, therefore another scan may be done after tilting the specimen. Moreover for anisotropic solids, the width of resonant peaks, required to infer damping, depends on combinations of complex moduli. ${ }^{28}$

Modes may be identified by repeating scans for different cube corner-orientations. ${ }^{29}$ Alternatively one may selectively excite classes of modes by exciting the specimen using eddy currents via a coil of wire surrounding the specimen and embedded in a magnetic field. ${ }^{30}$ One may also optically monitor the vibration using laser interferometry to identify the mode ${ }^{31,32}$ and avoid the need for a good initial guess of properties. If shear piezoelectric transducers ${ }^{33}$ are used rather than the usual compressional ones, one can identify some modes via the polarization of shear vibration; moreover, signal amplitude for the fundamental is enhanced by one to three orders of magnitude. One can perform RUS studies with off-the-shelf electronics, e.g., a function generator capable of sweeps, broadband ultrasonic transducers, and a digital oscilloscope. Lock-in amplifiers have been successfully used for RUS measurements, but it takes longer to perform a scan than with a heterodyne amplifier used in commercial RUS systems. ${ }^{34}$

If the specimen is isotropic, analytical formulas are available for the lowest mode. For example, ${ }^{35}$ the lowest mode for an isotropic cube is a torsional mode of frequency

$$
\nu=\frac{\sqrt{2}}{\pi L} \sqrt{\frac{G}{\rho}} .
$$

Here $L$ is the side length, $G$ is the shear modulus, and $\rho$ is the density. The next higher mode in the cube is about a factor of 
1.34 higher in frequency. For a cylinder of length $L$, the torsional natural frequencies for free-free vibration are

$$
\nu=\frac{n}{2 L} \sqrt{\frac{G}{\rho}}
$$

with $n$ a positive integer. The next higher mode is about a factor 1.26 higher. The fundamental torsion mode is the lowest mode for a cube and for a short cylinder of length equal to diameter if the Poisson's ratio is not too close to the lower limit -1 . The mode structure given is for an elastic material. For viscoelastic materials there is frequency dependence (dispersion) of the modulus. If $\tan \delta$ is relatively large, the dispersion may be sufficient to complicate the analysis. Numerical algorithms currently used do not account for dispersion, and are appropriate only for low damping anisotropic materials.

Material damping $\tan \delta$ is obtained from RUS data by calculating it from the width of resonance curves via Eq. (3). Higher modes are closely spaced. Overlap of broad resonant peaks in high damping materials renders it impractical to determine $\tan \delta$ above 0.01 to 0.02 at frequencies other than the fundamental. ${ }^{36}$

\section{E. Treatment of large and small phase}

For materials of low loss $\left(\tan \delta<10^{-4}\right.$ or equivalently $Q>10^{4}$ ), resonance methods are the most appropriate. Among resonance methods, it is easier to measure free decay of vibration than to tune a forced-vibration system through a very narrow resonant peak. The decay time of vibration in a high- $Q\left(Q \approx 10^{9}\right)$ resonator at acoustic or low ultrasonic frequency can be several hours long. A corresponding time is necessary to set up the vibration. During this period the frequency of the electrical source must be sufficiently stable to match that of the resonant specimen. It is necessary in these experiments to minimize all sources of parasitic damping. ${ }^{37}$ Indeed, single crystal sapphire and silicon at low temperature have been reported to exhibit $\tan \delta$ as small as $10^{-9}$. Parasitic energy dissipation due to air viscosity or radiation of sound into the air tends to become important for $\tan \delta$ $<10^{-4}$. Bending modes are more vulnerable to such error than torsion, and slender specimens more so than compact ones such as those used for RUS. Sound radiation errors for RUS $^{38}$ are more severe for compressional modes than torsional modes. To eliminate these errors, tests on low-loss materials are done under vacuum. Vibration energy can also leak out through the support of the specimen, and lead to an overestimate of $\tan \delta$. Such errors are minimized as follows. In fixed-free vibration, the support structure must be orders of magnitude more rigid than the specimen, otherwise parasitic dissipation from transmission of waves into the support will obtrude in the data. For a fiber specimen, the impedance mismatch between specimen and support is very favorable, allowing $\tan \delta$ as small as $10^{-6}$ to be measured as described below in Sec. V.

In free-free vibration, specimens are usually supported at a vibration node, where displacement is minimal. A fine thread used for support is sufficiently compliant that there is a large mismatch in mechanical impedance, hence minimal spurious energy loss. The thread is placed near a vibration node. Further reduction in parasitic damping is achieved by greasing the thread. ${ }^{39}$ If the support thread is to be used for driving or detection, it must be sufficiently far from the node. ${ }^{40}$ In bending experiments, it is better to have the bending motion in the horizontal direction than the vertical direction because less energy is lost in rotating the support thread than in stretching it. Measured quality factor $Q$ has been observed to vary with suspension length ${ }^{41}$ for low-damping columnar silicon. In one study, $Q$ measurements in fused silica $^{42}$ were limited by losses in suspension wires, as the measured $Q$ was found to depend on the shape of each resonant mode and the proximity of each mode's nodal regions to the suspension points. The highest $Q$ measured in this series was $1.9 \times 10^{7}$ at $26 \mathrm{kHz}$.

For low-loss materials a further matter to consider is the possibility of spurious energy loss from the transducers used to induce and detect vibration. Weak coupling is beneficial in this context. Capacitive transducers are favored because their coupling is intrinsically weak. Even so, it is wise to calculate the error caused by how much energy might be lost in the equivalent resistance of the transducer circuit through transducer coupling. Moreover, extra energy dissipation can occur in films adsorbed on the resonator as well as surface damage and defects. Consequently, the surface must be kept clean and free of damage if the intrinsic damping of the material is to be approached. Specimens are polished and annealed to reduce surface and internal defects. $Q$ values to $4 \times 10^{8}$ have been measured for sapphire at room temperature, and to 5 $\times 10^{9}$ at $4 \mathrm{~K}^{38}$

For high-loss materials $(\tan \delta>0.1)$, it is easy to measure the phase directly in the subresonant regime. In resonant experiments, the approximate formulas for half width and for free-decay are not applicable, and the more complex exact solution for the vibration must be used for analysis. For example, large damping can also be measured from resonantpeak width, but the analysis is more complicated than for low $\tan \delta$ because the exact solution is necessary. For example, exact relations between $Q$ and $\tan \delta$ require a model of the viscoelastic response of the material. ${ }^{43}$ If damping is independent of frequency then

$$
Q^{-1}=\sqrt{1+\tan \delta}-\sqrt{1-\tan \delta} .
$$

Moreover, resonant peaks in high-loss materials become broader and of lower amplitude, and signal strength is reduced; moreover, nearby modes in a distributed system may overlap.

As for the decrement $\Lambda$ in free-decay of vibration, recall for small $\delta, \Lambda \approx \pi \tan \delta$. The exact expression is ${ }^{44}$

$$
\tan \delta=[1-\exp (-2 \Lambda)] / 2 \pi
$$

in which these authors define the inverse quality factor $Q^{-1}$ in terms of a ratio of dissipated energy $\Delta W$ to stored energy $W$ in a cycle of deformation, equivalent to $\tan \delta$ in the symbols used here. (The authors use the symbol $\delta$ for the decrement.) The exact solution shows one cannot obtain the damping from the ratio of adjacent oscillations if $\tan \delta \geqslant 1 / 2 \pi$ $\approx 0.16$, because for such high damping there are no oscillations. 


\section{MICROSCALE AND NANOSCALE MEASUREMENTS}

Testing of materials on a small scale is done, as one might expect, by scaling down the size of the methods for force generation and deformation measurement.

As for fibers, at the scale of a few-hundred $\mu \mathrm{m}$ diameter, it is possible to use a miniature torsion pendulum based on electromagnetic generation of torque, and an optical-lever scheme (laser beam reflected from a mirror) to measure angular displacement. Such an approach has been used in the study of single osteon fibers (about $0.2 \mathrm{~mm}$ diameter) from bone. ${ }^{45}$ More slender fibers may be excited via a capacitive approach in which an oscillating voltage is applied to a plate within $1 \mathrm{~mm}$ of the fiber, and displacement measured by observing the shadow of the illuminated fiber with a split silicon-diode detector. ${ }^{46}$ The specimens were set into flexural vibration under vacuum. For silica fibers 0.23 to $0.5 \mathrm{~mm}$ in diameter in a fixed-free configuration, $\tan \delta$ of about $10^{-6}$ was measured in the frequency range $30 \mathrm{~Hz}$ to $1 \mathrm{kHz}$. The observed damping was comparable to predicted damping from thermoelastic coupling alone; therefore parasitic damping was minimal in this case.

Permanent magnets to generate torque or force can be scaled down to magnetic grains ${ }^{47,48}$ of micrometer-size or below. The motion of grains or beads ${ }^{49}$ embedded in a material may be examined by video microscopy or by lightscattering methods. As with macroscopic experiments, interpretation of results requires a quantitative model of the system to relate the raw force-displacement or torque-angle data to a stress-strain relationship. The embedded-bead approach has been used mostly for soft materials such as gels and biological cells. For such materials one need not necessarily excite the bead externally. Thermal motion of the bead may provide enough motion from which one can infer material properties. This approach has been used for membranes and other components of living cells as follows. A micrometer-size bead is embedded in the membrane and illuminated with an infrared laser. ${ }^{50}$ The laser light provides an optical trap. Material properties are inferred from the power spectrum of Brownian motion of the bead as determined from an image of the bead projected upon a split silicon-light sensor. The frequency range is from $100 \mathrm{~Hz}$ to several kilohertz.

Thin films may be applied to a substrate of known material, typically of low damping. The resulting laminate may be studied under quasistatic conditions or in lumped resonance. Film properties are extracted with the aid of composite-theory analysis of the laminate. One may also reflect ultrasonic waves from a surface with a thin film on it. ${ }^{51}$ Material properties are inferred from the amplitude and phase of the obliquely reflected waves. The approach is originally due to Mason et al. ${ }^{52}$ who studied viscoelastic properties of polymer liquids via oblique-wave reflection. Film properties can also be studied by a variant of resonant ultrasound spectroscopy in which a film of unknown material is applied to a block of material of known properties. ${ }^{53}$

As for properties of materials at and near the surface, indentation creep experiments offer potential for mapping properties or study of small samples. Nanoindentation creep has been conducted with a diamond tip upon polished specimens. ${ }^{54}$ Load was ramped to a steady value for creep tests. The tip was oscillated to obtain contact stiffness. No creep was seen in tungsten as one would expect at moderate stress levels and at room temperature. Scanning-probe microscopes can be configured to provide phase data as an alternative contrast variable in images. Thus far, the chain of inference has been too indirect to allow mapping of viscoelastic properties of hard material. The shape of the probe tip is often not known with great certainty, and adhesive and contact forces may complicate the analysis. ${ }^{50}$ Indeed, mapping of stiffness in a graphite-epoxy composite disclosed reasonable values of moduli ${ }^{55}$ but damping values for graphite were too high by orders of magnitude. Soft materials such as gels and biological materials have been studied with modified scanning-probe microscopes. Modification of the probe shape by polystyrene beads allows the experimenter to more readily infer material properties ${ }^{56}$ using the Hertz solution for a spherical contact problem. Moduli of several $\mathrm{kPa}$ and $\tan \delta$ of about 0.1 have been measured from 20 to $400 \mathrm{~Hz}$.

\section{FREQUENCY DOMAIN: WAVES}

\section{A. Shear and longitudinal waves}

Wave methods are appropriate at frequencies (typically megahertz to tens of megahertz) above those used in resonant and subresonant methods. Measurement of the wave speed allows one to infer a modulus. ${ }^{57}$ If the wavelength of a longitudinal wave is much longer than the specimen width or diameter, then the Poisson expansion and contraction is free to occur, and the wave speed (bar velocity $c_{B}$ ) is governed by Young's modulus $E$ and density $\rho$ :

$$
c_{B}=\sqrt{\frac{E}{\rho}} .
$$

If the wavelength of a longitudinal wave is much shorter than the specimen width or diameter, the Poisson effect is restrained. This is the typical situation for most ultrasonic studies at or above $1 \mathrm{MHz}$. The longitudinal wave speed $c_{L}$ is given by

$$
c_{L}=\sqrt{\frac{C_{1111}}{\rho}},
$$

with $C_{1111}$ as the tensorial modulus in the 1 direction. For isotropic materials ${ }^{58}$ we have

$$
C_{1111}=E \frac{1-\nu}{(1+\nu)(1-2 \nu)},
$$

with $\nu$ as Poisson's ratio. For wavelengths which are neither long nor short, the relationship, as one might expect, is more complicated. ${ }^{59}$

The speed $c_{T}$ of shear or transverse waves in an isotropic elastic medium is given by ${ }^{60}$

$$
c_{T}=\sqrt{\frac{G}{\rho}},
$$

with $G$ as the shear modulus. For viscoelastic materials, each modulus is interpreted as the absolute value of the complex 
modulus, e.g., $\left|G^{*}\right|$. Wave speed is determined from the time delay of a pulsed group of waves and the thickness of the specimen.

For ultrasonic waves (above the upper limit of the human ear, $20 \mathrm{kHz}$, and most commonly above $0.5 \mathrm{MHz}$ ) one uses piezoelectric transducers to generate and receive the waves. Ultrasonic measurements are commonly performed between 1 and $20 \mathrm{MHz}$, but it is possible by special methods to go to $1 \mathrm{GHz}$ and beyond. ${ }^{61,62}$ The electrical wave forms are usually bursts of several cycles at the frequency of interest. The bursts should be short enough in time to discriminate multiple echoes. The transducer may be coupled to the specimen by a thin layer of fluid such as water. This is sufficient if longitudinal waves are used but is not appropriate for shear waves because fluids do not sustain shear waves. The transducer may also be coupled with glue or highviscosity grease, particularly if quartz crystal plates are used as transducers. Wave velocity is determined from the time delay of the pulse and from the thickness of the specimen. The simplest approach is to find the time delay of the leading edge of the wave form, but that lacks precision because of the change of pulse shape as it propagates and the difficulty of identifying an equivalent equivalent point on two leading edges. A better approach is the pulse-echo-overlap method ${ }^{63}$ in which one controls an adjustable delay until the initial and final wave forms overlap. Precision of a few ppm can be achieved.

\section{B. Ultrasonic attenuation}

Wave amplitude decays with distance $\mathrm{d}$ because of geometrical spreading in three dimensions, as $1 / d^{2}$. If the waves are confined to one dimension, the amplitude also decays because of absorption in the material itself. This decay is called attenuation and is given the symbol $\alpha$. In a linear material, the amplitude decays with distance $\mathrm{d}$ as $\exp (-\alpha \mathrm{d})$. Attenuation is related to material damping $\delta$ as follows: ${ }^{64}$

$$
\alpha=\frac{\omega}{c} \tan \frac{\delta}{2} \text {. }
$$

One way to measure attenuation of ultrasonic waves is by comparing the signal amplitude transmitted through two samples of different length. ${ }^{65}$ The attenuation $\alpha$, in units of nepers per unit length is determined as follows from the magnitudes of the signals: $A_{1}$ through a specimen of length $L_{1}, A_{2}$ through a specimen of length $L_{2}$ :

$$
\alpha=\frac{\ln \left(A_{1} / A_{2}\right)}{\left(L_{1}-L_{2}\right)} .
$$

One neper is a decrease in amplitude of a factor of 1/e. Sometimes attenuation is expressed in terms of decibels $(\mathrm{dB})$, a logarithmic scale. In this approach it is necessary to be careful that the coupling between transducer and specimen is reproduced for both measurements. Coupling depends on the pressure applied to the transducer and the degree of flatness of the specimen surface.

Attenuation may be measured by examining the decay of amplitudes of echoes of a tone burst reverberating between parallel surfaces of a specimen. ${ }^{66}$ A single transducer may be used to transmit and to receive the waves. The inference of attenuation will be correct provided the energy lost into the transducer is small enough to neglect. One cannot use commercial nondestructive test transducers in this approach since they are designed to have maximal electromechanical coupling and they are damped to achieve well-defined pulses. Therefore energy lost into them is substantial. Quartz is favored as a transducer material for the echo method. Quartz has low damping and a relatively weak electromechanical coupling. Therefore little energy from the sound wave is converted back into electricity. Even so, the electric circuit attached to the transducer is usually designed to minimize losses, which are calculated to aid in interpreting the data. The quartz crystal plate may be glued to the specimen. If only longitudinal, not shear, waves will be used, the crystal may be coupled with a thin layer of oil or grease.

For small damping, hence small attenuation, one must consider the fact that wave propagation in real materials is three dimensional, not one dimensional. If the ultrasonic transducer is smaller than the specimen width the waves will diffract. Diffracted waves lost to the receiving transducer give rise to an overestimate of the true attenuation, therefore for low-damping materials, corrections for diffraction are applied. If the transducer is equal to or larger than the specimen width, internal reflections from the lateral surfaces may occur. Longitudinal waves can be converted to slower shear waves by such reflections.

For large damping, there is also considerable dispersion (frequency dependence) of the velocity, leading to distortion of the wave form. It may then be difficult to identify corresponding points on wave forms that have passed through different lengths of material. If that is a problem, one may resort to more complex methods based on Fourier transformation.

\section{METHODS FOR BROADBAND RESULTS}

\section{A. Motivation}

As we have seen, most methods provide results at a single frequency or over a relatively narrow domain, usually three decades or less, of time or frequency. A wider range is desirable because even a single-relaxation-time process, described by an exponential in the time domain, or a Debye peak in the frequency domain, occupies about one decade. Most viscoelastic properties occur over a broader range. In applications one may be interested in creep of a material as well as its ability to absorb vibration at acoustic frequency or waves at ultrasonic frequency.

\section{B. Time-temperature superposition}

Viscoelastic behavior depends on temperature. For example the relaxation modulus function may be written $E$ $=E(t, T)$ with $t$ as time and $T$ as temperature. The temperature dependence of properties can be exploited to extend the range of effective time or frequency of measured properties. Suppose a series of tests is done at different temperatures. One specimen may be used for each test, or one may allow adequate time for recovery between tests. Plot the test results versus $\log$ time or $\log$ frequency, depending on the type of 


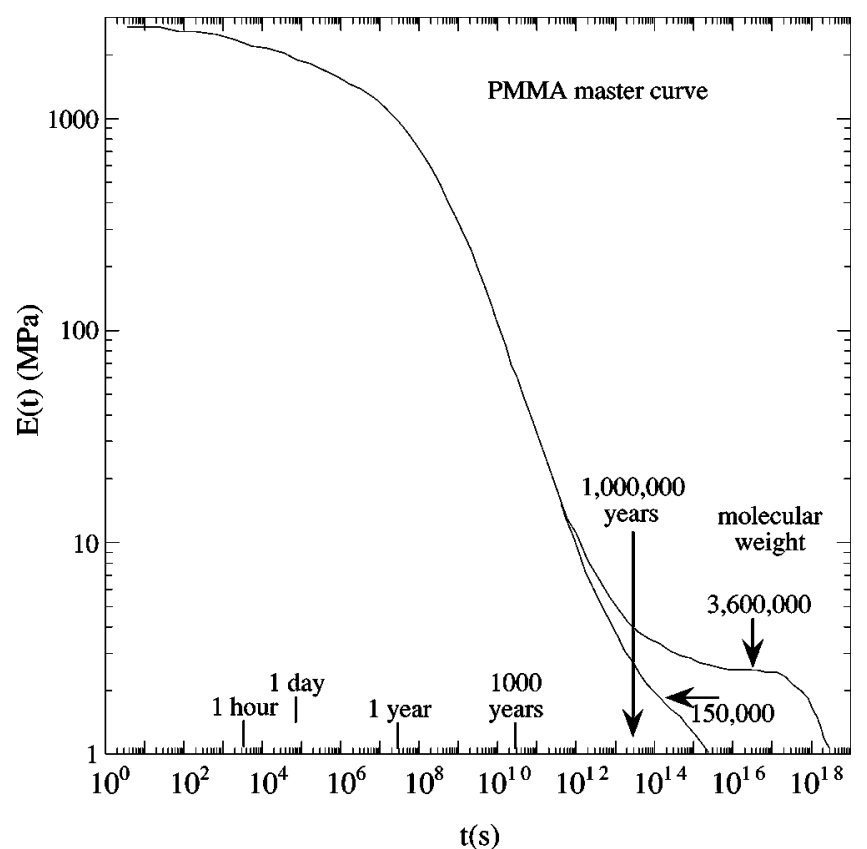

FIG. 12. Master curve for PMMA obtained from results at various temperatures via time-temperature superposition, adapted from McLoughlin and Tobolsky. ${ }^{10}$

test. It may be possible to shift the curves horizontally so that they coincide. If that is true, the material obeys timetemperature superposition. Such a shift is evidence that the temperature changes are effectively accelerating or retarding the dominant viscoelastic process or processes, because a shift on a $\log$ scale is equivalent to multiplying the scale by a number. The relaxation function is of the form

$$
E(t, T)=E\left(\zeta, T_{0}\right), \quad \text { with } \zeta=\frac{t}{a_{T}(T)},
$$

in which $\zeta$ is called the reduced time, $T_{0}$ is the reference temperature, and $a_{T}(T)$ is called the shift factor. The curve obtained by time-temperature shifts is called a master curve. Master curves covering 12 to 15 decades of time or frequency have been obtained for amorphous polymers. ${ }^{1} \mathrm{~A}$ representative master curve for polymethyl methacrylate (PMMA) is shown in Fig. 12.

Time-temperature superposition is commonly used in the study of polymers. It works well for many materials provided the widow of time or frequency is relatively narrow: three decades or less. The test of superposition is only definitive by its failure. ${ }^{67}$ Indeed, materials which appear to obey superposition over a three decade window are observed to deviate from it over a five or six decade window.

Time-temperature superposition will fail if the material undergoes a phase transformation in the temperature range of interest or if it is a composite with multiple processes which give rise to viscoelasticity, or if a dominant relaxation mechanism is not thermally activated.

Time-temperature superposition does not imply linear or nonlinear behavior. Conversely, a linearly viscoelastic material may or may not obey time-temperature superposition.

\section{Specific forms: Arrhenius and Williams, Landel, and Ferry (WLF)}

In some materials, the relaxation is dominated by a thermally activated ${ }^{2}$ process: the following Arrhenius equation applies:

$$
\nu=\nu_{0} \exp -\frac{U}{R T},
$$

with $U$ as the activation energy, $\nu$ as frequency for a feature such as a peak, $\nu_{0}$ as a frequency factor, and $R$ $=8.32 \mathrm{~J} / \mathrm{moleK}$ as the gas constant. One may use Boltzmann's constant $k=1.38 \times 10^{-23} \mathrm{~J} / \mathrm{K}$ if the energy is so expressed. For such materials, a change in temperature is equivalent to a shift of the behavior on the time or frequency axis. This is a particular form of time-temperature superposition, because a temperature change has the effect of stretching or shrinking the frequency scale. To use this approach, one may determine a series of temperature-dependent spectra at various frequencies, or a series of frequency-dependent spectra at a series of temperatures. ${ }^{68}$ If there is a peak at temperature $T_{p}$ one may plot $\ln \omega$ versus $1 / T_{p}$ to determine the activation energy. It is easier to obtain a reasonable range of frequency in subresonant tests than in resonant ones.

The Arrhenius condition has been used in the interpretation of experiments at a single frequency or a discrete set of frequencies. If the viscoelastic damping is in the form of a Debye peak with time constant $\tau_{0}$, the temperature at the peak $T_{p}$ follows ${ }^{18}$

$$
(U / k)\left(1 / T_{p}\right)=-\ln \omega \tau_{0} .
$$

In this case as well, the activation energy may be extracted from the slope $U / k$ of a plot of $\ln \omega$ versus $1 / T_{p}$, or a plot of $\log \omega$ versus $1 / T_{p}$ for which the slope is $U / 2.303 k$. When data are taken at only two frequencies in a temperature scan of a Debye peak, ${ }^{2}$

$$
\ln \left(\omega_{2} / \omega_{1}\right)=(U / k)\left(T_{1}^{-1}-T_{2}^{-1}\right) .
$$

The time-temperature shift for polymers tends to follow the empirical WLF equation (after Williams, Landel, and Ferry) $)^{1,69}$

$$
\ln \mathrm{a}_{\mathrm{T}}=-\frac{\mathrm{C}_{1}\left(\mathrm{~T}-\mathrm{T}_{\mathrm{ref}}\right)}{\mathrm{C}_{2}+\left(\mathrm{T}-\mathrm{T}_{\mathrm{ref}}\right)},
$$

in which $\mathrm{T}_{\text {ref }}$ is a reference temperature. The constants $C_{1}$ and $C_{2}$ depend on the particular polymer.

\section{Direct ways to achieve broadband results}

Direct measurements over a wide range of frequency are preferable to the more common indirect approach of varying the temperature, for the following reasons. ${ }^{3}$ Methods based on temperature shifts are restricted to activated processes, therefore viscoelastic spectra due to thermoelasticity or magnetic flux diffusion cannot be obtained by temperature scans. Second, the underlying viscoelastic theory is established for isothermal conditions and is not directly applicable if temperature is varied continuously. Finally, temperature variations can introduce important structural changes in the specimen during testing. 


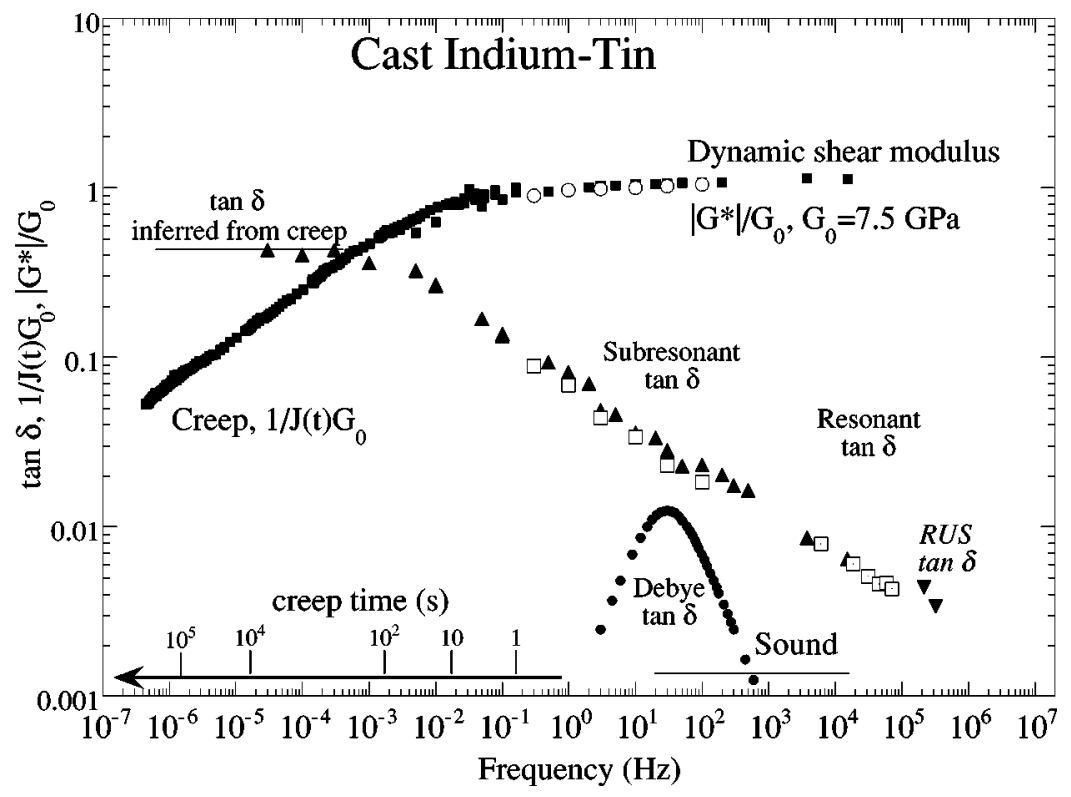

FIG. 13. Normalized modulus and $\tan \delta$ for indium-tin alloy ${ }^{37,75}$ at room temperature with no appeal to time temperature-superposition. Shown for comparison is the range of frequency perceived as sound by the ear, as well as a Debye peak in damping.

One may achieve a wide range of time or frequency at constant temperature by performing experiments with a variety of devices, in which each one covers a portion of the range. $^{70}$ This approach is cumbersome. Usually it is necessary to prepare several types of specimen. Six decades of frequency from $10^{-5}$ to $10 \mathrm{~Hz}$ are attainable via subresonant phase measurement as described ${ }^{3}$ in Sec. III B.

In general one can obtain an extended range of time and frequency in instruments which are capable of both transient and dynamic tests, and which have adequately fast short time/high frequency response. For example a commercial instrument or torsion pendulum, limited to $10 \mathrm{~Hz}$ (or $0.016 \mathrm{~s}$ ) by resonances in stalks and transducers, would provide six decades if the dynamic results were combined with results of about five hours of creep. Conversion of results from transient to dynamic or vice-versa may be done for linear materials by Fourier transformation or by approximate interrelations. For example, eight decades from $100 \mathrm{~Hz}$ down to one day of creep were achieved with a biaxial driven torsion pendulum $^{71}$ after modification. ${ }^{72}$ In this device, torque is generated via a moving coil immersed in a static magnetic field and is measured by a strain-gauge-type torque cell. Angular displacement is measured via a linear variable differential transformer coupled to a rotor. Ten decades from milliseconds to one year are achieved in broadband creep tests in which load is applied rapidly, and deformation measured electronically, ${ }^{73}$ but this requires considerable patience. An 11 decade range ${ }^{74}$ from $100 \mathrm{kHz}$ to subresonant tests in the $\mathrm{kHz}$ to $\mathrm{mHz}$ range to transient (creep) over several days was obtained as follows. Torque is applied to the specimen electromagnetically by a Helmholtz coil acting on a permanent magnet cemented to the end of the specimen. Deformation of the specimen end is determined by measurement of displacement of a laser beam using a split silicon-light sensor mounted upon a fast preamplifier. Resonances are eliminated from the torque-measuring and angle-measuring devices by this approach. Resonances in the specimen itself are analyzed via an analytical solution that is applicable to homogeneous cylindrical specimens, fixed at one end and free at the other, of any degree of loss. Higher harmonics in torsion are readily generated in this system. Representative results, compared with those of RUS, are shown in Fig. 13. In further development, noise in the $\tan \delta$ was reduced by using a lock-in amplifier. ${ }^{75}$

\section{DISCUSSION}

In this review, a variety of experimental methods for viscoelastic solids has been surveyed. Experimental methods are available over 20 decades of time and frequency. Most individual methods permit study at single frequencies or at most of three decades or less for a given specimen. If it is desired to conduct experiments over an extended range, one may use multiple instruments, time-temperature superposition (which is not appropriate for all materials), or careful design of a single instrument.

\section{ACKNOWLEDGMENTS}

The author acknowledges the support of the National Science Foundation through CMS-0136986 and the Materials Research Science and Engineering Center for Nanostructured Materials and Interfaces.

${ }^{1}$ J. D. Ferry, Viscoelastic Properties of Polymers, 2nd ed. (Wiley, New York, 1970).

${ }^{2}$ A. S. Nowick and B. S. Berry, Anelastic Relaxation in Crystalline Solids (Academic, New York, 1972).

${ }^{3}$ R. S. Lakes, Viscoelastic Solids (CRC Press, Boca Raton, FL, 1998).

${ }^{4}$ A. D. Nashif, D. I. G. Jones, and J. P. Henderson, Vibration Damping (Wiley, New York, 1985).

${ }^{5}$ S. Turner, The Physics of Glassy Polymers, edited by R. H. Haward (Wiley, New York, 1973), Chap. 4.

${ }^{6}$ K. Ninomiya and J. D. Ferry, J. Phys. Chem. 67, 2292 (1963).

${ }^{7}$ G. E. Troxell, J. M. Raphael, and R. W. Davis, ASTM Proc. 58, 1 (1958).

${ }^{8} \mathrm{H}$. Leaderman, Elastic and Creep Properties of Filamentous Materials and other High Polymers (Textile Foundation, Washington, DC, 1943).

${ }^{9}$ S. S. Sternstein and T. C. Ho, J. Appl. Phys. 43, 4370 (1972).

${ }^{10}$ J. R. McLoughlin and A. V. Tobolsky, J. Colloid Sci. 7, 555 (1952).

${ }^{11}$ A. Rivière, Mechanical Spectroscopy $Q^{-1} 2001$, edited by R. Schaller, G. Fantozzi, and G. Gremaud (Trans Tech Publications, Zurich, Switzerland, 2001), pp. 366-368. 
${ }^{12}$ S. S. Sternstein, Adv. Chem. Ser. 203, 123 (1983).

${ }^{13}$ T. S. Kê, Phys. Rev. 71, 533 (1947).

${ }^{14}$ J. Woirgard, Y. Sarrazin, and H. Chaumet, Rev. Sci. Instrum. 48, 1322 (1977).

${ }^{15}$ G. D’Anna and W. Benoit, Rev. Sci. Instrum. 61, 3821 (1990).

${ }^{16}$ P. Gadaud, B. Guisolan, A. Kulik, and R. Schaller, Rev. Sci. Instrum. 61, 2671 (1990).

${ }^{17}$ T. T. Gribb and R. F. Cooper, Rev. Sci. Instrum. 69, 559 (1998).

${ }^{18}$ I. G. Main, Vibrations and Waves in Physics (Cambridge, 1978).

${ }^{19}$ F. Förster, Z. Metallkd. 29, 109 (1937).

${ }^{20}$ P. Devos, R. De Batist, and J. Cornelis, Rev. Sci. Instrum. 65, 2819 (1994).

${ }^{21}$ B. E. Read and G. D. Dean, The Determination of Dynamic Properties of Polymers and Composites (Adam Hilger, Ltd., Bristol, England, 1978).

${ }^{22}$ S. L. Quimby, Phys. Rev. 25, 558 (1925).

${ }^{23}$ J. Marx, Rev. Sci. Instrum. 22, 503 (1951).

${ }^{24}$ W. H. Robinson, S. H. Carpenter, and J. L. Tallon, J. Appl. Phys. 45, 1975 (1974).

${ }^{25}$ J. Maynard, Phys. Today 49, 26 (1996).

${ }^{26}$ A. Migliori and J. L. Sarrao, Resonant Ultrasound Spectroscopy (Wiley, New York, 1997).

${ }^{27}$ R. G. Leisure and F. A. Willis, J. Phys.: Condens. Matter 9, 6001 (1997).

${ }^{28}$ Y. Sumino, I. Ohno, T. Goto, and M. Kumazawa, J. Phys. Earth 24, 263 (1976).

${ }^{29}$ A. Migliori, J. L. Sarrao, M. W. Visscher, T. Bell, M. Lei, Z. Fisk, and R. Leisure, Physica B 183, 1 (1993).

${ }^{30}$ H. Ogi, H. Ledbetter, S. Kim, and M. Hirao, J. Acoust. Soc. Am. 106, 660 (1999).

${ }^{31}$ H. Ogi, Y. Kawasaki, and M. Hirao, J. Appl. Phys. 92, 2451 (2002).

${ }^{32}$ H. Ogi, K. Sato, T. Asada, and M. Hirao, J. Acoust. Soc. Am. 112, 2553 (2002).

${ }^{33}$ Y. C. Wang and R. S. Lakes, Rev. Sci. Instrum. 74, 1371 (2003).

${ }^{34}$ A. Migliori, T. W. Darling, J. P. Baiardo, and F. Freibert, in Handbook of Elastic Properties of Solids, Liquids and Gases, edited by M. Levy, H. E. Bass, and R. R. Stern, Vol. 1 (Academic, New York, 2001), pp. 239-262.

${ }^{35}$ H. H. Demarest, Jr., J. Acoust. Soc. Am. 49, No. 3 (Part 2) 768 (1971).

${ }^{36}$ T. Lee, R. S. Lakes, and A. Lal, Rev. Sci. Instrum. 71, 2855 (2000).

${ }^{37}$ V. B. Braginskii, V. P. Mitrofanov, and V. I. Panov, Systems with Small Dissipation (University of Chicago Press, 1985).

${ }^{38}$ H. Zhang, R. S. Sorbello, C. Hucho, J. Herro, J. R. Feller, D. E. Beck, M. Levy, D. Isaak, J. D. Carnes, and O. Anderson, J. Acoust. Soc. Am. 103, 2385 (1998).

${ }^{39}$ J. E. Bishop and V. K. Kinra, $M^{3} D$ : Mechanics and Mechanisms of Material Damping, ASTM STP 1169, edited by V. K. Kinra and A. Wolfenden (ASTM, Philadelphia, PA, 1992).

${ }^{40}$ J. H. Wachtman, Jr. and W. E. Tefft, Rev. Sci. Instrum. 29, 517 (1958).

${ }^{41}$ J. E. Logan, N. A. Robertson, and J. Hough, Phys. Lett. A 170, 352 (1992).

${ }^{42}$ W. J. Startin, M. A. Beilby, and P. R. Saulson, Rev. Sci. Instrum. 69, 3681 (1998).

${ }^{43}$ E. J. Graesser and C. R. Wong, in $M^{3} D$ : Mechanics and Mechanisms of Material Damping, edited by V. K. Kinra and A. Wolfenden (ASTM, Philadelphia, PA, 1992), Vol. ASTM STP 1169.
${ }^{44}$ X. Zhu, J. Shui, and J. S. Williams, Rev. Sci. Instrum. 68, 3116 (1997).

${ }^{45}$ P. Frasca, R. A. Harper, and J. L. Katz, J. Biomed. Eng. 103, 146 (1981).

${ }^{46}$ J. Kovalik and P. R. Saulson, Rev. Sci. Instrum. 64, 2942 (1993).

${ }^{47}$ H. Freundlich and W. Seifritz, Z. Phys. Chem. (Munich) 104, 233 (1922).

${ }^{48}$ F. Amblard, B. Yurke, A. Pargellis, and S. Leibler, Rev. Sci. Instrum. 67, 818 (1996).

${ }^{49}$ F. C. MacKintosh and C. F. Schmidt, Curr. Opin. Colloid Interface Sci. 4, 300 (1999).

${ }^{50}$ E. Helfer, S. Harlepp, L. Bourdieu, J. Robert, F. C. MacKintosh, and D. Chatenay, Phys. Rev. Lett. 85, 457 (2000).

${ }^{51}$ I. Alig, D. Lellinger, J. Sulimma, and S. Tadjbakhsch, Rev. Sci. Instrum. 68, 1536 (1997).

${ }^{52}$ W. P. Mason, W. O. Baker, H. J. Mcskimin, and J. H. Heiss, Phys. Rev. 75, 936 (1949).

${ }^{53}$ H. Ogi, G. Shimoike, and M. Hirao, J. Appl. Phys. 91, 4857 (2002).

${ }^{54}$ A. Syed Asif and J. B. Pethica, Philos. Mag. A 76, 1105 (1997).

${ }^{55}$ S. A. Syed Asif, K. J. Wahl, R. J. Colton, and O. L. Warren, J. Appl. Phys. 90, 1192 (2001).

${ }^{56}$ R. E. Mahaffy, C. K. Shih, F. C. MacKintosh, and J. Käs, Phys. Rev. Lett. 85, 880 (2000).

${ }^{57}$ J. Kolsky, Philos. Mag. 1, 693 (1957).

${ }^{58}$ I. S. Sokolnikoff, Mathematical Theory of Elasticity (Krieger; Malabar, FL, 1983).

${ }^{59}$ D. Bancroft, Phys. Rev. 59, 588 (1941).

${ }^{60}$ H. J. McSkimin, in Physical Acoustics, edited by E. P. Mason, Vol. 1A, 271 (1964).

${ }^{61}$ G. Srinivasan, G. Wu, and T. T. Srinivasan, J. Appl. Phys. 67, 5463 (1990).

${ }^{62}$ R. Homer, G. C. Alexandrakis, and G. Dewar, J. Appl. Phys. 61, 4133 (1987).

${ }^{63}$ E. Papadakis and T. P. Leach, in Handbook of Elastic Properties of Solids, Liquids and Gases, edited by M. Levy, H. E. Bass, and R. R. Stern, Vol. 1 (Academic, New York, 2001), Chap. 2, pp. 39-66.

${ }^{64}$ R. M. Christensen, Theory of Viscoelasticity (Academic, New York, 1982).

${ }^{65}$ R. Truel, C. Elbaum, and B. Chick, Ultrasonic Methods in Solid State Physics (Academic, New York, 1966).

${ }^{66}$ E. Papadakis, in Physical Acoustics, edited by R. N. Thurston and A. D. Pierce, Vol. XIX (Academic, New York, 1990), pp. 107-155.

${ }^{67}$ D. J. Plazek, J. Rheol. 40, 987 (1996).

${ }^{68}$ J. San Juan, "Mechanical Spectroscopy", in Mechanical Spectroscopy $Q^{-1}$ 2001, edited by R. Schaller, G. Fantozzi, and G. Gremaud (Trans Tech Publications, Zurich, Switzerland, 2001), pp. 32-73.

${ }^{69}$ M. L. William, R. F. Landel, and J. D. Ferry, J. Am. Chem. Soc. 77, 3701 (1955).

${ }^{70}$ V. J. Koppelmann, Kolloid-Z. 144, 12 (1955).

${ }^{71}$ F. A. Myers, F. C. Cama, and S. S. Sternstein, Ann. N.Y. Acad. Sci. 279, 94 (1976).

${ }^{72}$ R. S. Lakes, J. L. Katz, and S. S. Sternstein, J. Biomech. 12, 657 (1979).

${ }^{73}$ W. Letherisch, Br. J. Appl. Phys. 1, 294 (1950).

${ }^{74}$ M. Brodt, L. S. Cook, and R. S. Lakes, Rev. Sci. Instrum. 66, 5292 (1995).

${ }^{75}$ P. M. Buechner, D. Stone, and R. S. Lakes, Scr. Mater. 41, 561 (1999).

${ }^{76}$ T. S. Kê, J. Appl. Phys. 19, 285 (1948). 